\title{
Review
}

\section{Emerging Potential Role of Autophagy to Modulate Aggresome Formation: insights Molecular Treatment of Alzheimer's Disease}

\author{
Md. Ataur Rahman ${ }^{1,2 *}$, MD. Hasanur Rahman ${ }^{3}$, ANM Mamun-Or-Rashid ${ }^{4}$, Hongik Hwang ${ }^{1}$, \\ Hyewhon Rhim ${ }^{1,5 *}$
}

${ }^{1}$ Center for Neuroscience, Brain Science Institute, Korea Institute of Science and Technology (KIST), Seoul 02792, Republic of Korea.

${ }^{2}$ Global Biotechnology \& Biomedical Research Network (GBBRN), Department of Biotechnology and Genetic Engineering, Faculty of Biological Sciences, Islamic University, Kushtia 7003, Bangladesh.

${ }^{3}$ Department of Biotechnology and Genetic Engineering, Bangabandhu Sheikh Mujibur Rahman Science and Technology University, Gopalganj-8100, Bangladesh.

${ }^{4}$ Anti-Aging Medical Research Center and Glycation Stress Research Center, Graduate School of Life and Medical Sciences, Doshisha University, Kyoto 602-8566, Japan.

${ }^{5}$ Division of Bio-Medical Science and Technology, KIST School, Korea University of Science and Technology (UST), Seoul 02792, Republic of Korea.

*Correspondence address to: Center for Neuroscience, Brain Science Institute, Korea Institute of Science and Technology (KIST), 5 Hwarang-ro 14-gil, Seongbuk-gu, Seoul 02792, Republic of Korea. Md. Ataur Rahman, E-mail: ataur1981rahman@hotmail.com; Hyewhon Rhim, Tel.: +822-958-5923, Fax: +82-2- 958-6937, E-mail: hrhim@kist.re.kr 


\begin{abstract}
Alzheimer's disease (AD) is one of the most prevailing neurodegenerative diseases in the world, which is characterized by memory dysfunction and the formation of tau and amyloid $\beta$ (A $\beta$ ) aggregate in multiple brain regions, including the hippocampus and cortex. The formation of senile plaques involving tau hyperphosphorylation, fibrillar A $\beta$, and neurofibrillary tangles (NFTs) are used as pathological markers of $\mathrm{AD}$, and eventually produces aggregation or misfolded protein. Importantly, it has been found that failure to degrade these aggregate-prone proteins leads to pathological consequences, such as synaptic impairment, cytotoxicity, neuronal atrophy, and memory deficits associated with AD. Recently, increasing evidences have been suggested that autophagy pathway plays a role as a central cellular protection system to prevent the toxicity induced by aggregate or misfolded proteins. Moreover, it has also been related that AD-related protein aggresomes could be selectively degraded by autophagosome and lysosomal fusion through autophagy pathway which is known as aggrephagy. Therefore, the regulation of autophagy might be served as a useful approach to modulate the formation of aggresome associated in AD. This review focuses on the recent improvements in the application of natural compounds and small molecules as a potential therapeutic approach for AD prevention and treatment via aggrephagy.
\end{abstract}

Key words: Alzheimer's disease (AD); aggregation; autophagy; aggresome; autophagosomes; aggrephagy. 


\section{Introduction}

Aggresomes are inclusion bodies consist of aggregated cytoplasmic proteins induced by the overexpression or inhibition of certain proteins of the proteasome system [1], and the accumulation of incorrectly folded proteins is thought to contribute to the etiology of various neurodegenerative diseases [2]. Misfolded protein molecules processing has an important to maintain normal cellular function as well as homeostasis. There are three protein quality systems have been found to degrade misfolded or aggregated protein, such as ubiquitinated proteasomal degradation, chaperone-mediated degradation, and selective autophagy or aggrephagy [3]. Previous studies have been demonstrated that an increasing trend in the number of AD-related proteins are associated with aggresome [4], and non-pathological proteins could form invasive inclusion bodies as well. It has been found that silencing the expression of ubiquitin ligase HRD1 in SH-SY5Y human neuroblastoma cells prevents the formation of APP [5], and ubiquitin 1 linked to AD is known to regulate presenilin-1, which plays a key role of invasiveness in AD pathogenesis [6]. It has been hypothesized that autophagy manipulations might be a potentially promising therapeutic strategies target to modulate protein aggregation-related diseases and toxicity.

In this review, we would like to emphasize the susceptibility of AD-associated proteins to autophagy. New evidences have been suggested that macrolysis (henceforth mentioned as autophagy) of the hemolysate-mediated degradation system is the main regulator of internal kinetics [7]. For instance, independent reports have demonstrated the significance of autophagy in neurodegenerative diseases and inclusion body formation [8,9]. Furthermore, in other studies, the pharmacological initiation of autophagy has promoted the breakdown of aggregated proteins, such as Huntington's protein (Htt) and tau mutants, while increasing cell viability [10,11], thus suggesting that targeting the autophagy pathway may provide innovative methods for treating diseases related to protein conformational changes. Attack formation promotes the delivery of decentralized proteins aggregation to the autophagic pathway [12]. Pharmacological inhibition of autophagy delayed the elimination of the attack formed by the mutant peripheral myelin protein PMP22, a Schwann cell protein associated with demyelinating neuropathy [13]. It has been exhibited that aggresomes produced by the Htt mutant positively stained certain proteins related to autophagy and sensitive to eliminate by autophagy [14]. However, it has not been determined whether autophagy easily eliminates invasion caused by other proteins associated with the disease. 


\section{Mechanism of aggresome formation}

Aggrephagy is a selective form of autophagy that eliminates aggregated and ubiquitinated proteins [15]. The first stage of aggresome formation involves the accumulation of irregularly folded or unfolded proteins, packed into larger insoluble aggregates and transported to the microtubuleorganizing center (MTOC) [15,16], and aggresomes can cause autophagic degradation. In autophagic process, autophagosomes undergo lysosomal degradation through dual autophagy membranes encompassing ubiquitin-labeled proteins $[17,18]$. The autophagy pathway plays an important role in the cytoplasm, but it is not efficient in the nucleus because autophagy-related molecules do not have nuclear inclusions. Aggresomes are membrane-free microtubule-dependent cytoplasmic inclusion bodies that form oligomer complexes with amorphous structure containing unfolded or misfolded proteins which are generally stable and insoluble under physiological conditions [19,20]. Typically, aggregated proteins continue to grow and develop, and then oligomerize to form longer insoluble inclusions bodies or aggregates [21]. These aggregates can either link with themselves or join with existing aggresomes located at the MTOC, residing near the cellular centrosome. These aggregates are labeled with ubiquitin alone with heat shock proteins (Hsps), such as Hsp70, and their development in neurodegenerative diseases is demonstrated in Figure 1 [3,22],[23,24]. Also, aggresomes are primarily surrounded by the cytoskeleton of intermediate filament, and a cage-like structure appears after collapsing with cytoskeleton, such as vimentin and keratin, along with neurofilaments [19,25] (Figure 1). This cage-like structure is known to promote structural stability and inhibit non-specific interactions. These aggresomes exert cellular cytotoxicity, but at a later stage, matured bilayer autophagosomes lose their activity [26]. The components of the ubiquitin-proteasome systems are concentrated or grouped into these assemblies before discarding of aggregates molecules through degradation or refolding [27]. Recently, it has been reported that the modulation of aggresome formation and assembly may serve as a novel approach to treat diseases associated with a defect in protein conformation. 


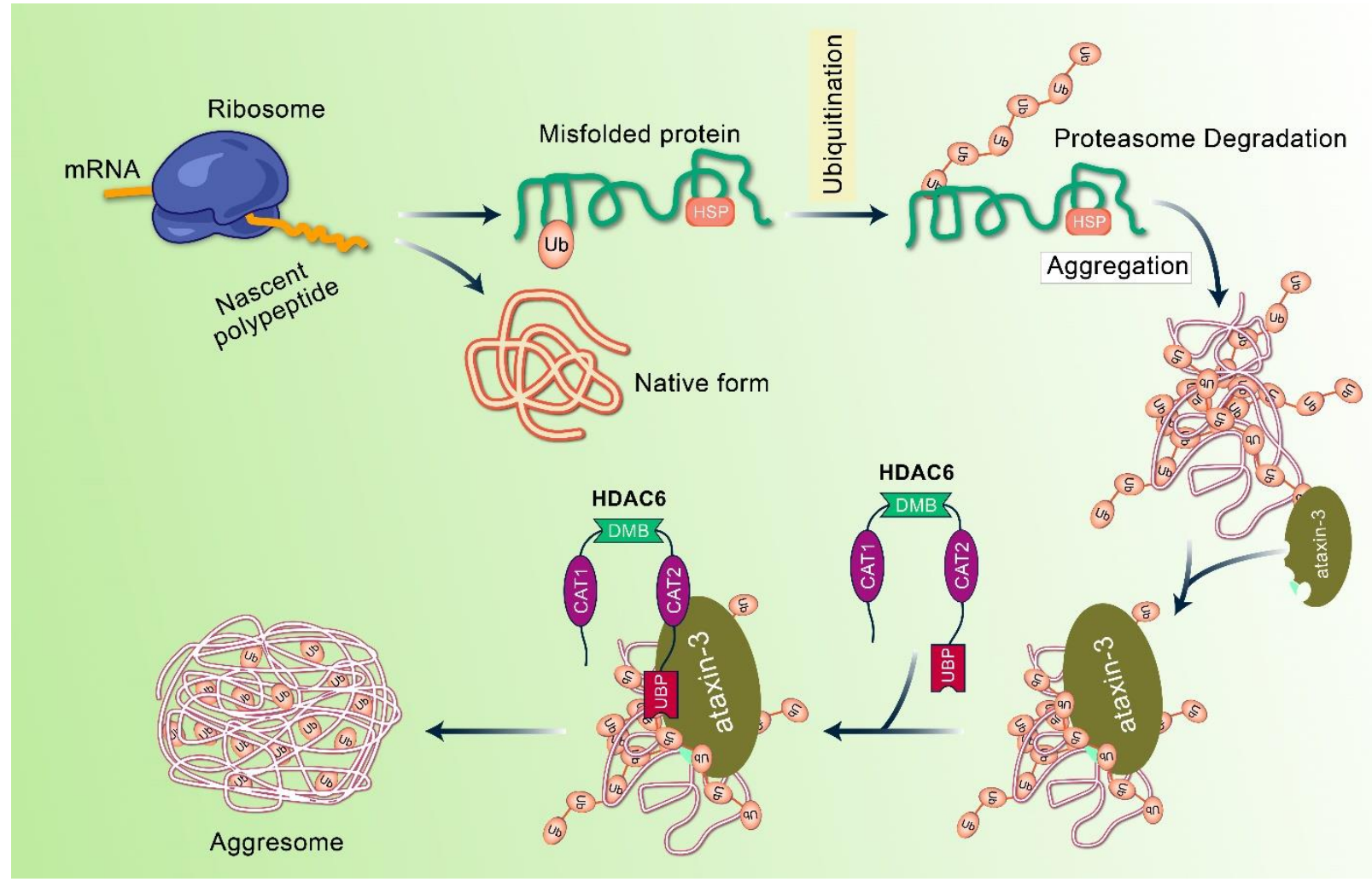

Figure 1: Molecular mechanism of aggresome development and formation. Under normal conditions, misfolded and polyubiquitinated proteins are fragmented via the ubiquitin proteasomal system. When ubiquitin proteasomal system is altered or overhauled, misfolded polyubiquitin proteins accumulate and form to aggregate. In this case, ataxin-3-dexiquitinase interrelates and aggregates with polyubiquitinated proteins to form ubiquitin chains structure. In addition, HDAC6 binds these non-anchored $\mathrm{C}$-terminal tails of ubiquitin to form aggregates and recruits them into the dynein motor complex.

\section{Clearance of aggresomes through autophagy}

When the proteasomal degradation system is overwhelmed, the autophagy pathway is activated as an essential cellular defense system to resist incorrect folding and to prevent the accumulation of aggregated proteins $[2,28]$. In this system, poorly folded and aggregated proteins are selectively identified and transferred to the MTOC (center of microtubule tissue) around the central body by microtubule-based and microtubule-based retrograde transport [29]. Accumulating evidences suggest that autophagy not only protects aggressive cells by chelating poorly folded cytotoxic aggregates, but also enriches poorly folded aggregates for subsequent removal [12]. Autophagy is 
a multi-step progression well-characterized by the construction of an insulating membrane, named phagophore, which swells to form the double-membrane autophagosome and fuses with the lysosome to degrade the sequestered cytoplasmic cargo [28,30]. Unlike proteasomes, autophagy does not require substrate expansion, and is capable of degrading large protein complexes, protein aggregates, and even entire organelles [31,32]. Moreover, autophagy is a precisely controlled process involving multiple proteins encoded by the Atg (autophagy-related) genes [33,34], and previous studies revealed that autophagy is induced in response to the oxidative stress or proteasomal damage, and is directly involved in the elimination of aggresomes [35,36].

Emerging evidences suggest that autophagy is responsible for the removal of aggregated and misfolded proteins, and the inhibition of autophagy preferentially affects the degradation of mutant proteins associated with neurodegenerative diseases while leaving their wild-type counterparts unaffected [37]. Although the mechanism of autophagy for the specific elimination of misfolded proteins is not yet clear at the molecular level, selective isolation of incorrectly folded proteins in aggresomes can facilitate the preferential removal of abnormal autophagy proteins [38]. By chelating the endogenous autophagy inhibitor mTOR (mammalian target of rapamycin) kinase [39], the aggresome can also contribute to the initiation of autophagy [40,41]. Importantly, the conventional concept of autophagy only involves the non-selective removal of misfolded proteins and regular cellular proteins, whereas the aggresome-autophagy pathway is specific to aggregated and misfolded proteins. Therefore, a special type of autophagy induction is thought to be related to the specific elimination of toxicity linked to the aggresome formation. One potential mechanism has been found in parkin-mediated Lys $^{63}$-linked polyubiquitination which promotes the elimination of misfolded proteins via autophagy, and autophagy occurs by binding to the adapter protein p62 [42], an Ub binding protein interacting with the ubiquitinated proteins via the autophagic mechanical component LC3 through its UBA domain (connected to Ub) and the 22 amino acid LIR (LC3 interaction region) [43]. p62 promotes the binding of a polyubiquitin chain linked to Lys63 [30,31], and the inhibition of its UBA or LIR domain changes the conditions of the ubiquitinated aggregates in the autophagosomes $[44,45]$. According to recent evidences, p62 may also promote the formation of protein aggregates in addition to promoting the elimination of autophagy [46]. Further studies are required to determine whether p62 is indeed an Ub receptor, 
which regulates the processing of Lys63-linked folded polyubiquitinated proteins via an active aggresome-autophagy pathway [47] (Figure 2).

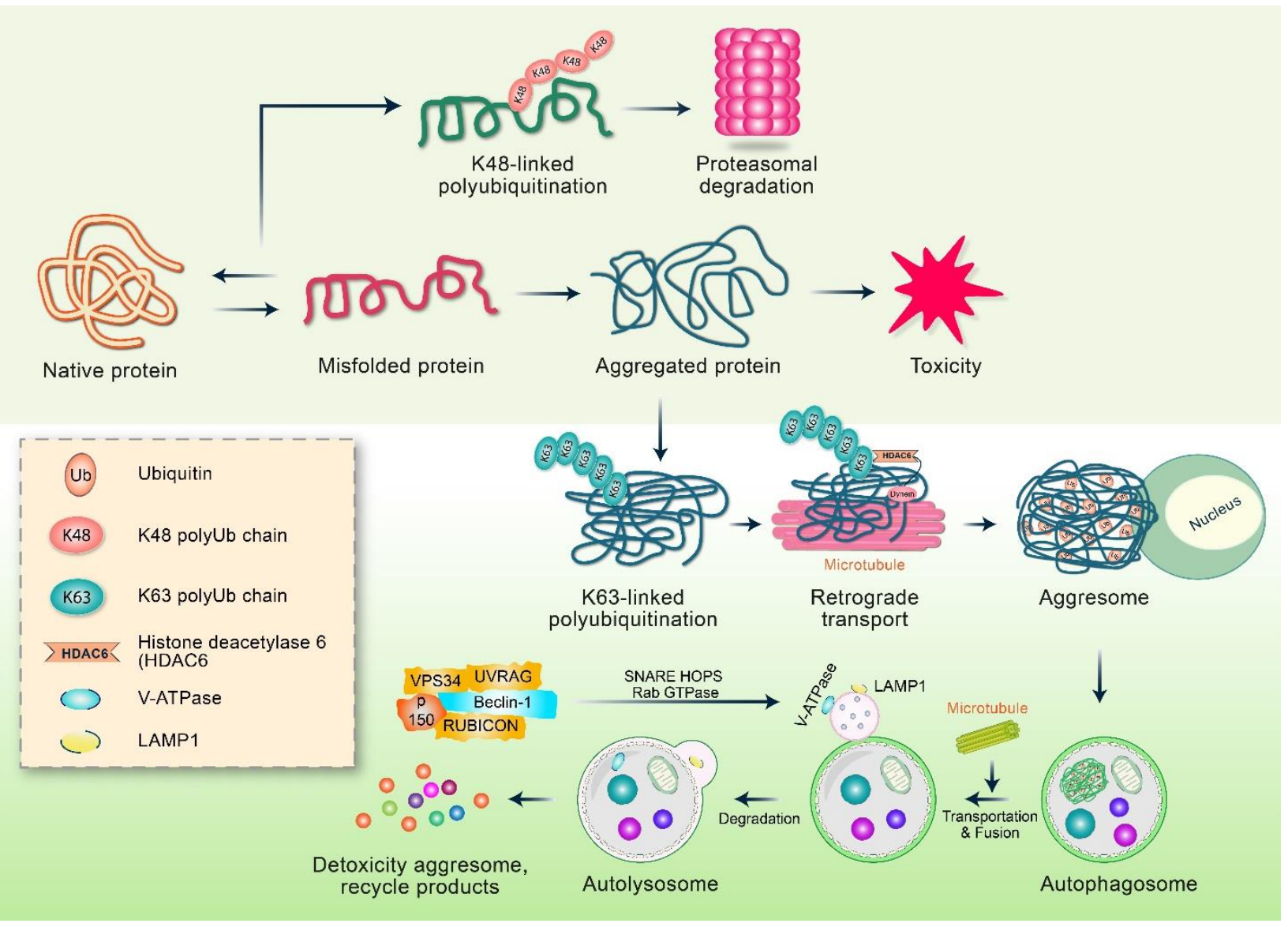

Figure 2: The regulation of aggresome-autophagy pathway. Aggresome and autophagy are regulated by Lys63-associated polyquitination mediated pathway. Oxidative damage or genetic mutations are responsible to cause protein folded. After folding, the misfolded proteins are labeled with polyubiquitin chains linked to Lys48, which is subsequently degraded via either the proteasomal system or chaperones-mediated pathway. However, when proteasome and chaperone systems are overwhelmed, misfolded proteins form oligomers or aggregates with cellular toxicity. Moreover, PD-related parkin ligase E3 acts with the E2 enzymes Ubc13/Uev1a to facilitate Lys63associated polyubiquitination of misfolded proteins in proteasome injury conditions. Polyubiquitin chain stimulates the binding with HDAC6 and the misfolded proteins bind to dynein motor complex which retrograde transports aggresome to MTOC. Polyubiquitination encourages p62 binding in addition to recruiting autophagic membranes to form autophagosomes. Consequently, 
the fusion of autophagosomes and lysosomes facilitates the degradation of misfolded and aggregated proteins through the lysosomal hydrolases.

\section{Molecular mechanism of the fusion of aggresome and lysosome}

In the final stage of degradation, fully matured and closed membrane aggresome fuses with lysosomes to form hybrid compartment organelles called "autolysosomes" which digests their contents [16] (Figure 2). Although the fusion process is morphologically characterized well, its mechanism remains relatively elusive at the molecular level. Typically, the separation membrane is fused with the lysosomal membrane, and then the lysosomal hydrolase breaks down the content of the fused (or aggresome/lysosome) autolysosome. The substance (amino acid, etc.) is eventually degraded, which is then either recycled or removed into extracellular space [48]. Subsequently, the lysosomal fraction of the autolysosomes is recovered to produce new lysosomes [49]. Rab7A (Ypt7 in yeast), mature autophagosome, HOPS (homologous protein fusion and classification complex) and SNARE receptor (SNAP (soluble adhesion protein NSF)) (NSF, fusion-sensitive) labeled (ethyl-maleimide) proteins are essential for the fusion of autophagosomes and lysosomes [48]. The SNAREs increases the permeability of the membrane, forming the membrane opening and fusing the content of two adjacent organelles [50]. The Rab7A is transformed into an active form linked to GTP through the action of HOPS (Figure 2). The fusion of organelle binding is dependent on the complex of Rab7A and HOPS (Rab7A downstream effector), which is present in two membranes fused, and SNARE and Rab7A are enriched at the fusion site [51]. SNAREs are also required for the expansion and closure of autophagosomal membranes, the early stages of autophagy, and for the transportation of Atg9 (a transmembrane protein necessary for membrane development) [52]. Recently, it has been found that the SNARE protein syntaxin 17 (STX17) is employed in the outer membrane of the autophagosomes and mediates the lysosomal fusion $[53,54]$. The depletion of STX17 prevents the destruction of autolysosomes and leads to the accumulation of autophagosomes in the process of basal and starvation-induced autophagy [55]. It is currently unknown whether STX17 is also involved in selective aggrephagy [56]. Furthermore, when autophagosomal membrane is formed around the materials to be submerged in the endoplasmic reticulum, STX17 interacts with the autophagosome-labeled Atg14L protein at a very early stage [57,58]. These findings together suggest that STX17 plays an important role at both ends of the autophagy decomposition pathway. 


\section{The role of autophagy to modulate the aggregation in AD}

The crucial function of autophagy in preserving neuronal homeostasis is well established in in vivo studies conducted in mice, in which the inhibition of autophagy results in protein deposition and neurodegeneration over time [59]. Decreased autophagy is related to an increase in age-associated neurodegenerative diseases, such as AD [60]. Defective autophagy typically involves decreased expression of major receptors for autophagy, and altered orientation substrate and autophagosome formation. Emphasis has been placed on several aspects, such as the lack of maturation, degradation and lysosomal alteration [61]. Interestingly, several genes involved in protein homeostasis in neurodegenerative diseases are mutated, such as the lysosome-autophagy system and ubiquitin-proteasome system [62]. In the cases of neurodegenerative diseases, the first marker of altered autophagy is abnormal quantity of autophagosomes or amphisomes, which can lead to the generation of ROS and other cytotoxic elements. Incidentally, the accumulation of autophagosomes is characterized by an endogenous pool of amyloidogenic A $\beta$ peptides in animal models with AD [63].

The potential function of SQSTM1/p62 in AD progression and other neurodegenerative disorders has been receiving more attention [64]. Compared to the control group, cytoplasmic SQSTM1/p62 protein levels were found to be reduced in the frontal cortex of $\mathrm{AD}$ patients $[65,66]$, indicating that SQSTM1/p62 expression is downregulated and the protein is retained in the pellet [66]. Interestingly, oxidative impairment to the promoter of SQSTM1/p62 leads to the reduction in gene transcription, which also appeared in the brain of AD patients [66]. Histopathological analysis of hippocampal and cortical samples from patients with AD showed that the SQSTM1/p62 protein is contained in inclusion bodies mainly consisted of phosphorylated tau, TRAF6 (tumor necrosis factor receptor associated 6), and ubiquitin, promoting the formation of aggresome [67]. Moreover, co-localization of SQSTM1/p62 and Keap-1 insoluble deposits was observed in AD brain extracts [68], and the impairment to the promoter of SQSTM1/p62 as well as co-localization of SQSTM1/p62 with protein aggregation have been observed in other neuropathies, such as Huntington's disease, tauopathy and $\alpha$-synucleinopathies $[64,66]$. Several evidences suggested that SQSTM1/p62 plays a central role in the transport of tau protein to proteasome [67], and that there is a negative connection between the levels of SQSTM1/p62 and p-tau [69], indicating that reduced SQSTM1/p62 function likely causes the accumulation of tau positive aggregates with age. Notably, 
a SQSTM1/p62-deficient mouse model has typical AD phenotype, including p-tau neurofibrillary tangles, memory impairment, and synaptic depletion [70]. In addition to protein homeostasis, these last two effects are consistent with the assumption that autophagy is necessary for cell remodeling and neuronal plasticity, and autophagy is a prerequisite for neurogenesis and memory processes [71]. However, it is unknown whether a change in the levels of SQSTM1/p62 is correlated with the elimination of abnormal aggresomes in AD patients. Collectively, current findings together suggest that it would be promising to decrease the formation of aggresomes in $\mathrm{AD}$ via modulating autophagy.

\section{Potential therapeutic action of autophagy to control aggresome formation in AD pathogenesis}

Natural compounds or small molecules have been used to induce clearance of aggresome and to restore or enhance cognitive function in patients with AD [4]. For example, liraglutide has been found to activate insulin degradation enzyme (IDE), increase cognition function and long term potentiation (LTP), and reduce $A \beta$ plaque deposition and inflammation in APP/PS1 mice via mTOR-independent and JNK pathway [72,73]. Also, rapamycin, a well-known natural macrolide, was found to mitigate $A \beta$ plaques, liberate cerebral amyloid angiopathy, and enhance memory impairment in the AD mice model of PDAPP, hAPP (J20) and P301S through the inhibition of mTOR activity [74,75]. Moreover, a polyphenolic compound known as curcumin, a PI3K/mTOR inhibitor, not only relieved $\mathrm{AD}$ pathology by decreasing $\mathrm{A} \beta$ but also repaired spatial memory function in APP/PS1 mice through the degradation of autophagic A $\beta$ aggregates [76]. In clinical trials using JNPL3 mice as an AD model, autophagy induced by methylene blue was shown to decrease the aggregation insoluble tau through the inhibition of mTOR [77]. Another polyphenol, oleuropein aglycone, also promotes autophagy by releasing $\mathrm{Ca}^{2+}$ from reticulum and preventing the activity of mTOR in TgCRND8 mice, and considerably downregulated A $\beta$ plaque [78]. A carbazole-based fluorophore, SLM, binds to $\mathrm{A} \beta$ and prevents $\mathrm{A} \beta$ aggregation in $3 \times \mathrm{Tg}$-AD mouse model by alleviating the pathological and behavioral impairments in AD [79]. Additionally, human prolactin-releasing peptide palmitoylated analog, palm11-PrRP31, was shown to protect neuronal cells in APP/PS1 mice model of AD and decrease astrogliosis, microgliosis, $\beta$-amyloid plaque load as well. [80]. The list of representative natural compounds and small molecules that modulate aggresome formation in AD pathogenesis by enhancing autophagy is summarized in Table 1. 
Table 1: Natural compounds and small molecules that modulate autophagic activity and control aggresome formation in AD models.

\begin{tabular}{|c|c|c|c|c|}
\hline $\begin{array}{c}\text { Natural } \\
\text { compounds/ small } \\
\text { molecules }\end{array}$ & AD model & $\begin{array}{l}\text { Molecular } \\
\text { mechanism }\end{array}$ & Research outcomes & References \\
\hline Fisetin & $\begin{array}{l}\text { Mouse and } \\
\text { rat primary } \\
\text { cortical } \\
\text { neurons }\end{array}$ & $\begin{array}{l}\text { mTOR inhibition, } \\
\text { TFEB and Nrf2 } \\
\text { activation }\end{array}$ & $\begin{array}{c}\text { Autophagy induction, Decrease } \\
\text { sarkosyl-insoluble tau } \\
\text { phosphorylation }\end{array}$ & [81] \\
\hline Ouabain & $\begin{array}{c}\text { Tau } \\
\text { transgenic } \\
\text { fly, P301L } \\
\text { mice }\end{array}$ & $\begin{array}{c}\text { Inactivation of } \\
\text { mTOR, } \\
\text { Activation of TFEB }\end{array}$ & $\begin{array}{c}\text { Increase autophagy, } \\
\text { Decrease toxic tau, } \\
\text { Increase memory function }\end{array}$ & [82] \\
\hline $\begin{array}{l}\text { SLM, a carbazole- } \\
\text { based fluorophore }\end{array}$ & $3 x T g-A D$ & $\begin{array}{c}\text { Activating GSK- } \\
3 \beta \text {, Reduces } \\
\text { neuroinflammation }\end{array}$ & $\begin{array}{c}\text { Decreasing } \mathrm{A} \beta 40 \text { and } \mathrm{A} \beta 42 \\
\text { levels, } \\
\text { Reducing phosphorylation of tau }\end{array}$ & [79] \\
\hline Aspirin & $5 \mathrm{xFAD}$ & $\begin{array}{c}\text { Activation of } \\
\text { PPAR } \alpha \text { and TFEB }\end{array}$ & $\begin{array}{c}\text { Increase lysosomal biogenesis, } \\
\text { Decrease } A \beta\end{array}$ & [83] \\
\hline Liraglutide & $\begin{array}{c}\text { APP/PS1, } \\
\text { APPswe/SH- } \\
\text { SY5Y cells }\end{array}$ & $\begin{array}{c}\text { Increase IDE } \\
\text { levels, } \\
\text { mTOR- } \\
\text { independent, JNK } \\
\text { activation }\end{array}$ & $\begin{array}{l}\text { Improved cognitive function, } \\
\text { Reduced A } \beta \text { plaque deposition } \\
\text { and inflammation, } \\
\text { Enhanced LTP, Autophagy } \\
\text { activation }\end{array}$ & {$[72,73]$} \\
\hline Rapamycin & $\begin{array}{l}\text { Transgenic } \\
\text { (h)APP mice }\end{array}$ & mTOR inactivation & $\begin{array}{c}\text { Improved memory, Decrease } \\
\text { sarkosyl-insoluble tau, }\end{array}$ & {$[74,75]$} \\
\hline Cinnamic acid & $5 \times \mathrm{xFAD}$ & $\begin{array}{l}\text { Activates PPAR } \alpha, \\
\text { Upregulate TFEB }\end{array}$ & $\begin{array}{l}\text { Reduced cerebral A } \beta \text { plaque } \\
\text { burden, Improved memory } \\
\text { function, Stimulate lysosomal } \\
\text { biogenesis. }\end{array}$ & [84] \\
\hline Trehalose & $\begin{array}{l}\text { APP/PS1, } \\
\text { Tg2576 }\end{array}$ & $\begin{array}{c}\text { Increase } \\
\text { synaptophysin, } \\
\text { doublecortin, and } \\
\text { progranulin }\end{array}$ & $\begin{array}{c}\text { Inhibits tau, Improve cognitive } \\
\text { and learning ability }\end{array}$ & {$[85,86]$} \\
\hline Curcumin & APP/PS1 & mTOR inactivation & $\begin{array}{c}\text { Reduced } \mathrm{A} \beta \text { plaque, Increase } \\
\text { memory function }\end{array}$ & [76] \\
\hline
\end{tabular}




\begin{tabular}{|c|c|c|c|c|}
\hline Oleuropein aglycone & $\begin{array}{c}\text { TgCRND8 } \\
\text { mice }\end{array}$ & $\begin{array}{c}\text { Inhibition mTOR } \\
\text { and } \mathrm{Ca}^{2+} \text { liberating }\end{array}$ & $\begin{array}{c}\text { Reduced A } \beta \text { plaque, Increase } \\
\text { synaptic plasticity }\end{array}$ & {$[78]$} \\
\hline Hep-14 & APP/PS1 & Upregulate TFEB & Reduction of A $\beta$ plaque & {$[87]$} \\
\hline Palm ${ }^{11}$-PrRP31 & APP/PS1 & $\begin{array}{c}\text { Activation of pre- } \\
\text { synaptic marker } \\
\text { synaptophysin, }\end{array}$ & $\begin{array}{c}\text { Decrease in Tau phosphorylation, } \\
\text { Reduce A } \beta \text { plaque and } \\
\text { microgliosis, }\end{array}$ & {$[80]$} \\
\hline Methylene blue & JNPL3 & mTOR inactivation & $\begin{array}{c}\text { Reduces insoluble tau, Increases } \\
\text { memory function. }\end{array}$ & {$[77]$} \\
\hline Temsirolimus & P301S mice & Inhibition of \\
& mTOR & $\begin{array}{c}\text { Improve motor amd memory } \\
\text { function, Sarkosyl insoluble tau } \\
\text { reduction, }\end{array}$ & {$[88]$} \\
\hline
\end{tabular}

Recently, mTOR-independent transcription factor, TFEB, was shown to reduce aggresome formation and activate autophagy, and several compounds are capable of stimulating TFEBinduced lysosomal biogenesis and mTOR-independent autophagy (Figure 3). For example, ouabain has been found to activate TFEB, decrease the aggregation of p-Tau, and increase cognition function in P301L mice [82]. Cinnamic acid and aspirin are found to activate TFEB promotor and promotes lysosomal biogenesis which decreases the formation of $A \beta$ plaque in 5xFAD mice model $[83,84]$. Trehalose, a disaccharide molecule, activates calcineurin and protein phosphatase-3 CB (PP3CB) by promoting the translocation of TFEB into the nucleus via mTORindependent autophagy [85]. Additionally, it has been found that, in APP/PS1 mice, trehalose treatment promotes the clearance of $A \beta$ plaque independent of the mTOR pathway $[85,86]$. Hep14, a cardiac glycoside-ingenol, increases TFEB-induced ALP by activating PKC and inhibiting GSK3 $\beta$, thus decreasing plaques formation in APP/PS1 AD mice model [87]. Jiang et al. has demonstrated that the treatment of temsirolimus successfully improved autophagic clearance of hyperphosphorylated tau in the brain of P301S transgenic mice and okadaic acid-incubated SHSY5Y cells. In addition, temsirolimus administration improved memory impairments and spatial learning function in P301S mice [88]. Small molecule or natural compounds may control autophagy-lysosomal process via mTOR- and TFEB-mediated pathways, which is summarized in Figure 3. 


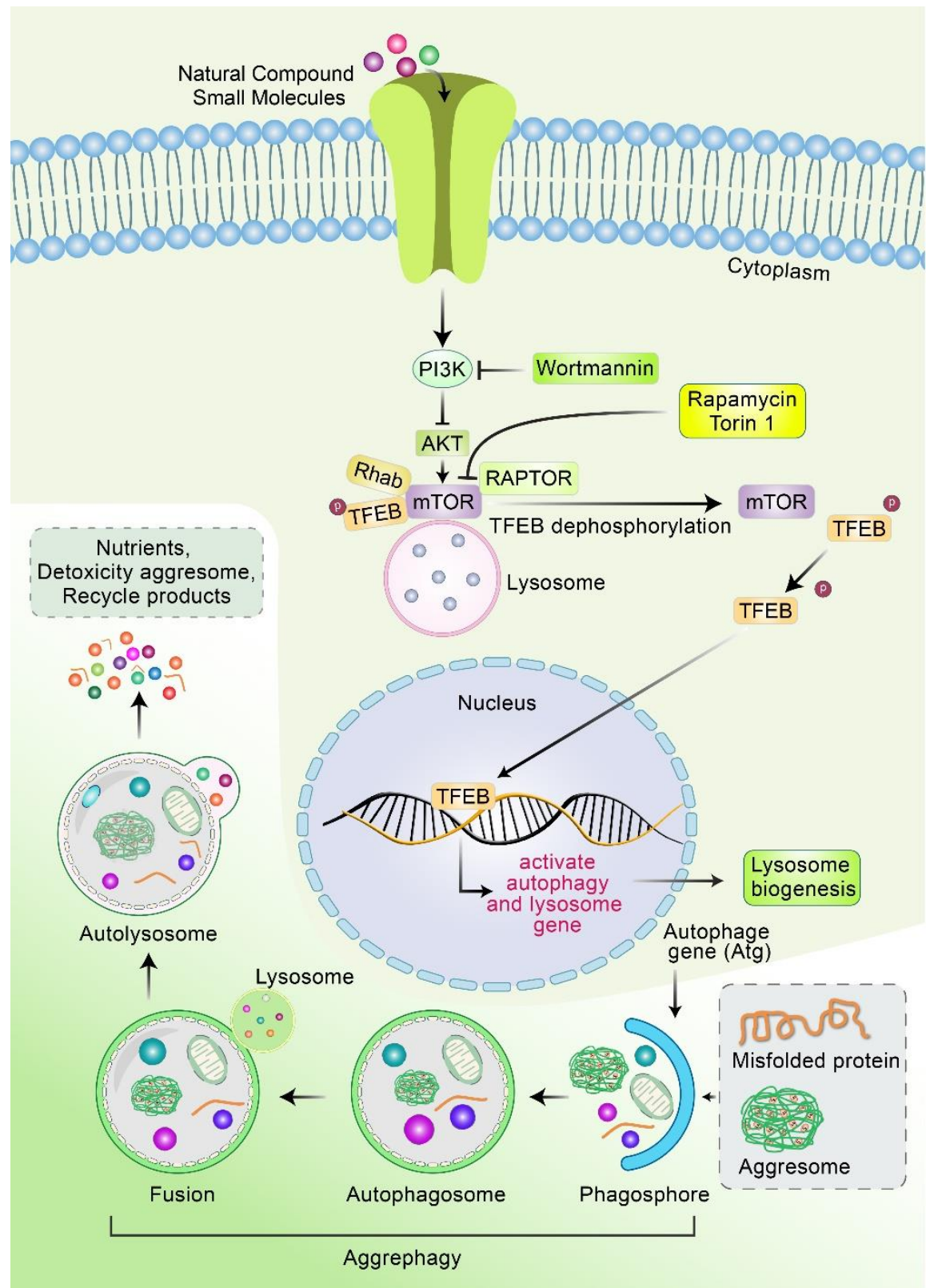

Figure 3: Mechanism of natural compounds or small molecules to activate mTOR and TFEB in autophagy-lysosomal process. Natural compounds or small molecules inactivate AKT and mTOR, which promotes the accumulation of TFEB in the cytoplasmic and its nuclear translocation. TFEB in cytoplasm is heavily phosphorylated and interacts with mTOR in the lysosome surface. The inactivation of mTOR activity stimulates the dephosphorylation of TFEB. Subsequently, dephosphorylated TFEB is translocated from cytoplasm to nucleus. In nucleus, TFEB binds to the promoter regions of autophagy- and lysosomal-associated genes and induces gene expression in 
addition to lysosome biogenesis. Aggresome is bound to phagosphore, resulting in the formation of autophagosome. Eventually, autophagosome fused with lysosome degrades aggresome via aggrephagy process.

\section{Future prospective of inhibiting aggresome formation as a treatment for AD}

Recently, extensive resources were dedicated to the development of high-performance, automated detection platforms to identify compounds that can prevent aggresome development and endorse aggresome formation [89]. Such high-performance screening primarily uses large libraries designed for general-purpose detection, which serves as an useful approach when running screens with unknown targets or with no structural information available $[89,90]$. These screenings play an important role in drug discovery and the creation of new forms of chemical treatment $[89,90]$. However, since protein aggregation and the formation of aggresomes are complex and mediated by multi-step processes [91], special care must be taken in interpreting the results, especially when aggresome-related genes are used as reporter genes. For example, inhibiting the early stage of autophagy pathway reduces the formation of aggresome and the levels of toxic protein species [92] (Figure 4). However, although preventing the late stages of autophagic pathway reduces aggresome development, it was shown to increase the assembly of soluble-toxic protein species. This complexity highlights the importance of verifying and characterizing the target sites as well as working mechanisms of candidate compounds. 


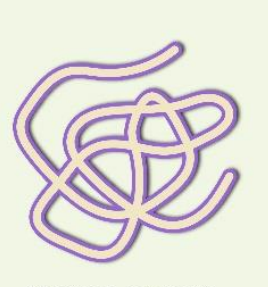

Native protein

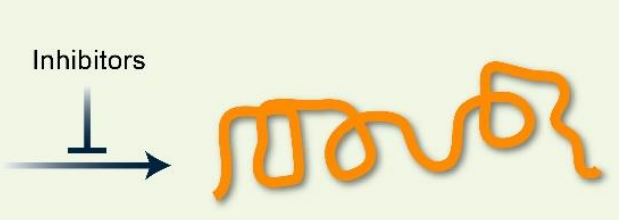

Misfolded protein

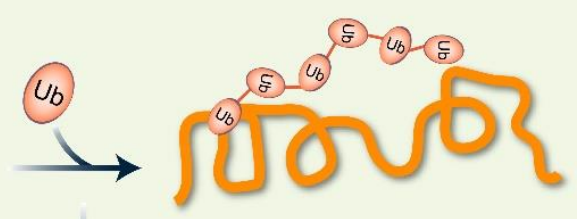

Polyubiquitinqted protein

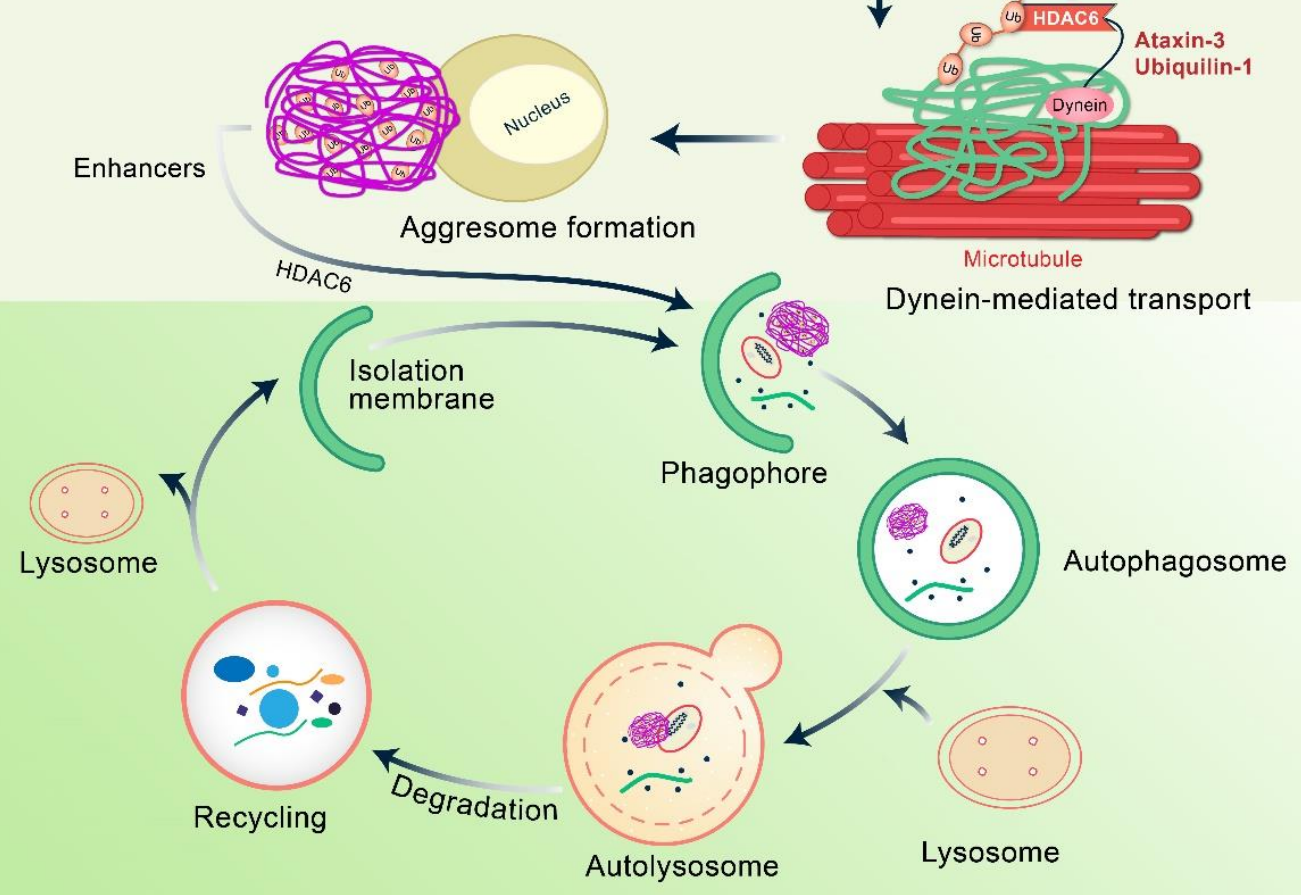

Figure 4: Regulation of misfolded protein by autophagy. Misfolded proteins are recognized and polyubiquitinated by ubiquitin E3 protein ligases. Adapter proteins, such as HDAC6, ataxin3 and ubiquitin-1, bind to polyubiquitinated proteins on the dynein motor complex for retrograde transport to the aggressor. Aggresome takes over the autophagy mechanism, including HDAC6, and breaks down aggresome. Several steps along this path enable small molecules to block incorrect protein folding and improve the coupling of folded proteins to dynein for retrograde transport, or improve the clearance of aggresome by autophagy, which can be potentially applied in the treatment of neurodegenerative diseases.

\section{Conclusions}

Autophagy enhancers that can be utilized for the potential treatment of neurodegenerative diseases is receiving growing attention in recent studies $[9,18,93,94]$. It has been shown that the inactivation of mTOR by the lipophilic macrolide antibiotic rapamycin promotes the induction of autophagy 
[95]. Furthermore, long-term administration of rapamycin can reduce amyloid load in mouse models of $\mathrm{AD}$ and improve cognitive function as well as the pathology of tauopathy [96,97]. In addition, post-translational modifications, such as ubiquitination, acetylation, O-GlcNAcylation, and phosphorylation also appear to exert a positive effect on autophagy [45,98]. Acetylation is an important cellular mechanism that protects cells from stress stimuli, and can be changed in neurodegenerative pathologies, highlighting acetylation and deacetylation processes as a potential therapeutic candidate for neurodegenerative diseases. Several selective small molecule inhibitors of HDAC6 have been identified [99]. For example, tubastatin A promotes the acetylation of $\alpha$ tubulin, stabilizes the microtubule network, and confers neuroprotection on neurons during in vitro culture and neurodegeneration. By clarifying the mechanism underlying the selection of lysosomal loads, autophagy-based approach will serve as a more effective therapeutic candidate [100]. In the case of $\mathrm{AD}$, aggresome formation can occur, which can induce the inhibition of proteasome activity. Aggrephagy and its downstream signaling cascades offer promising new therapeutic targets for preventing $\mathrm{AD}$, and further research is required to clarify the relationship between the mechanisms involved in autophagic activities and the formation of aggresomes.

\section{Authors' Contributions}

MAR designed and proposed the original idea of this manuscript. MHR prepared the figures and ANMMR wrote the draft. HH reviewed the manuscript. HR reviewed the scientific contents of the manuscript. All authors read and approved the final version of this manuscript.

\section{Funding:}

This work was funded by NRF Research Program (2016M3C7A1913845) and supported by the Korea Research Fellowship (KRF) Program (2016H1D3A1908615) through the National Research Foundation of Korea, Ministry of Science and ICT, Republic of Korea.

Conflict of Interest: The authors declare that they have no conflict of interest. 


\section{References}

[1] J.A. Johnston, C.L. Ward, R.R. Kopito, Aggresomes: a cellular response to misfolded proteins, J Cell Biol 143 (1998) 1883-1898. 10.1083/jcb.143.7.1883.

[2] M. Takalo, A. Salminen, H. Soininen, M. Hiltunen, A. Haapasalo, Protein aggregation and degradation mechanisms in neurodegenerative diseases, Am J Neurodegener Dis 2 (2013) 1-14.

[3] J.M.T. Hyttinen, M. Amadio, J. Viiri, A. Pascale, A. Salminen, K. Kaarniranta, Clearance of misfolded and aggregated proteins by aggrephagy and implications for aggregation diseases, Ageing Research Reviews 18 (2014) 16-28. 10.1016/j.arr.2014.07.002.

[4] S. Malampati, J.X. Song, B.C.K. Tong, A. Nalluri, C.B. Yang, Z.Y. Wang, S.G. Sreenivasmurthy, Z. Zhu, J. Liu, C.F. Su, S. Krishnamoorthi, A. Iyaswamy, K.H. Cheung, J.H. Lu, M. Li, Targeting Aggrephagy for the Treatment of Alzheimer's Disease, Cells-Basel 9 (2020). ARTN 311

10.3390/cells9020311.

[5] M. Kaneko, H. Koike, R. Saito, Y. Kitamura, Y. Okuma, Y. Nomura, Loss of HRD1-mediated protein degradation causes amyloid precursor protein accumulation and amyloid-beta generation, J Neurosci 30 (2010) 3924-3932. 10.1523/JNEUROSCI.2422-09.2010.

[6] S. Zhang, M. Zhang, F. Cai, W. Song, Biological function of Presenilin and its role in AD pathogenesis, Transl Neurodegener 2 (2013) 15. 10.1186/2047-9158-2-15.

[7] T. Nagao, M. Hirokawa, Diagnosis and treatment of macrocytic anemias in adults, J Gen Fam Med 18 (2017) 200-204. 10.1002/jgf2.31.

[8] M. Kim, A. Ho, J.H. Lee, Autophagy and Human Neurodegenerative Diseases-A Fly's Perspective, Int J Mol Sci 18 (2017). 10.3390/ijms18071596.

[9] F. Guo, X. Liu, H. Cai, W. Le, Autophagy in neurodegenerative diseases: pathogenesis and therapy, Brain Pathol 28 (2018) 3-13. 10.1111/bpa.12545.

[10] B. Boland, W.H. Yu, O. Corti, B. Mollereau, A. Henriques, E. Bezard, G.M. Pastores, D.C. Rubinsztein, R.A. Nixon, M.R. Duchen, G.R. Mallucci, G. Kroemer-, B. Levine, E.L. Eskelinen, F. Mochel, M. Spedding, C. Louis, O.R. Martin, M.J. Millan, Promoting the clearance of neurotoxic proteins in neurodegenerative disorders of ageing, Nat Rev Drug Discov 17 (2018) 660-+. 10.1038/nrd.2018.109.

[11] A.M. Palhegyi, E. Seranova, S. Dimova, S. Hoque, S. Sarkar, Biomedical Implications of Autophagy in Macromolecule Storage Disorders, Front Cell Dev Biol 7 (2019). UNSP 179

10.3389/fcell.2019.00179.

[12] P. Sweeney, H. Park, M. Baumann, J. Dunlop, J. Frydman, R. Kopito, A. McCampbell, G. Leblanc, A. Venkateswaran, A. Nurmi, R. Hodgson, Protein misfolding in neurodegenerative diseases: implications and strategies, Translational Neurodegeneration 6 (2017). ARTN 6

10.1186/s40035-017-0077-5.

[13] J.A. Gomez-Sanchez, L. Carty, M. Iruarrizaga-Lejarreta, M. Palomo-Irigoyen, M. Varela-Rey, M. Griffith, J. Hantke, N. Macias-Camara, M. Azkargorta, I. Aurrekoetxea, V.G. De Juan, H.B.J. Jefferies, P. Aspichueta, F. Elortza, A.M. Aransay, M.L. Martinez-Chantar, F. Baas, J.M. Mato, R. Mirsky, A. Woodhoo, K.R. Jessen, Schwann cell autophagy, myelinophagy, initiates myelin clearance from injured nerves, Journal of Cell Biology 210 (2015) 153-168. 10.1083/jcb.201503019.

[14] S. Waelter, A. Boeddrich, R. Lurz, E. Scherzinger, G. Lueder, H. Lehrach, E.E. Wanker, Accumulation of mutant huntingtin fragments in aggresome-like inclusion bodies as a result of insufficient protein degradation, Mol Biol Cell 12 (2001) 1393-1407. DOI 10.1091/mbc.12.5.1393.

[15] T. Lamark, T. Johansen, Aggrephagy: selective disposal of protein aggregates by macroautophagy, Int J Cell Biol 2012 (2012) 736905. 10.1155/2012/736905. 
[16] J.M. Hyttinen, M. Amadio, J. Viiri, A. Pascale, A. Salminen, K. Kaarniranta, Clearance of misfolded and aggregated proteins by aggrephagy and implications for aggregation diseases, Ageing Res Rev 18 (2014) 16-28. 10.1016/j.arr.2014.07.002.

[17] J. Fortun, W.A. Dunn, Jr., S. Joy, J. Li, L. Notterpek, Emerging role for autophagy in the removal of aggresomes in Schwann cells, J Neurosci 23 (2003) 10672-10680.

[18] M.A. Rahman, H. Rhim, Therapeutic implication of autophagy in neurodegenerative diseases, BMB Rep 50 (2017) 345-354. 10.5483/bmbrep.2017.50.7.069.

[19] R.R. Kopito, Aggresomes, inclusion bodies and protein aggregation, Trends Cell Biol 10 (2000) 524530. Doi 10.1016/S0962-8924(00)01852-3.

[20] K.A. Markossian, B.I. Kurganov, Protein folding, misfolding, and aggregation. formation of inclusion bodies and aggresomes, Biochemistry-Moscow+ $69 \quad$ (2004) 971-984. DOI 10.1023/B:BIRY.0000043539.07961.4c.

[21] S. Ventura, Sequence determinants of protein aggregation: tools to increase protein solubility, Microb Cell Fact 4 (2005). Artn 11

10.1186/1475-2859-4-11.

[22] H. Ouyang, Y.O. Ali, M. Ravichandran, A. Dong, W. Qiu, F. MacKenzie, S. Dhe-Paganon, C.H. Arrowsmith, R.G. Zhai, Protein aggregates are recruited to aggresome by histone deacetylase 6 via unanchored ubiquitin C termini, J Biol Chem 287 (2012) 2317-2327. 10.1074/jbc.M111.273730.

[23] R.C. Lu, M.S. Tan, H. Wang, A.M. Xie, J.T. Yu, L. Tan, Heat Shock Protein 70 in Alzheimer's Disease, Biomed Res Int 2014 (2014). Artn 435203

$10.1155 / 2014 / 435203$.

[24] Z. Xu, K. Graham, M. Foote, F.S. Liang, R. Rizkallah, M. Hurt, Y.C. Wang, Y.Y. Wu, Y. Zhou, 14-3-3 protein targets misfolded chaperone-associated proteins to aggresomes, J Cell Sci 126 (2013) 4173-4186. $10.1242 /$ jcs.126102.

[25] R. Heir, C. Ablasou, E. Dumontier, M. Elliott, C. Fagotto-Kaufmann, F.K. Bedford, The UBL domain of PLIC-1 regulates aggresome formation, Embo Rep 7 (2006) 1252-1258. 10.1038/sj.embor.7400823.

[26] I. Dikic, Z. Elazar, Mechanism and medical implications of mammalian autophagy, Nat Rev Mol Cell Biol 19 (2018) 349-364. 10.1038/s41580-018-0003-4.

[27] R.H. Chen, Y.H. Chen, T.Y. Huang, Ubiquitin-mediated regulation of autophagy, J Biomed Sci 26 (2019) 80. 10.1186/s12929-019-0569-y.

[28] M.A. Rahman, S.K. Saha, M.S. Rahman, M.J. Uddin, M.S. Uddin, M.G. Pang, H. Rhim, S.G. Cho, Molecular Insights Into Therapeutic Potential of Autophagy Modulation by Natural Products for Cancer Stem Cells, Front Cell Dev Biol 8 (2020). ARTN 283

10.3389/fcell.2020.00283.

[29] A.D. Sanchez, J.L. Feldman, Microtubule-organizing centers: from the centrosome to noncentrosomal sites, Curr Opin Cell Biol 44 (2017) 93-101. 10.1016/j.ceb.2016.09.003.

[30] M.A. Rahman, M.R. Rahman, T. Zaman, M.S. Uddin, R. Islam, M.M. Abdel-Daim, H. Rhim, Emerging Potential of Naturally Occurring Autophagy Modulators Against Neurodegeneration, Curr Pharm Design 26 (2020) 772-779. 10.2174/1381612826666200107142541.

[31] M.A. Rahman, H. Hwang, S.Y. Nah, H. Rhim, Gintonin stimulates autophagic flux in primary cortical astrocytes, J Ginseng Res 44 (2020) 67-78. 10.1016/j.jgr.2018.08.004.

[32] W.W.Y. Yim, N. Mizushima, Lysosome biology in autophagy, Cell Discov 6 (2020). ARTN 6

10.1038/s41421-020-0141-7. 
[33] X.H. Li, S.K. He, B.Y. Ma, Autophagy and autophagy-related proteins in cancer, Mol Cancer 19 (2020). ARTN 12

10.1186/s12943-020-1138-4.

[34] M.A. Rahman, K. Bishayee, K. Habib, A. Sadra, S.O. Huh, 18alpha-Glycyrrhetinic acid lethality for neuroblastoma cells via de-regulating the Beclin-1/Bcl-2 complex and inducing apoptosis, Biochem Pharmacol 117 (2016) 97-112. 10.1016/j.bcp.2016.08.006.

[35] N.M. Kocaturk, D. Gozuacik, Crosstalk Between Mammalian Autophagy and the UbiquitinProteasome System, Front Cell Dev Biol 6 (2018). UNSP 128

10.3389/fcell.2018.00128.

[36] M.S. Uddin, A. Al Abdullah, M.A. Rahman, M.T. Kabir, S. Alkahtani, I.S. Alanazi, A. Perveen, G.M. Ashraf, M.N. Bin-Jumah, M.M. Abdel-Daim, Exploring the Promise of Flavonoids to Combat Neuropathic Pain: From Molecular Mechanisms to Therapeutic Implications, Front Neurosci-Switz 14 (2020). ARTN 478

10.3389/fnins.2020.00478.

[37] N. Peker, D. Gozuacik, Autophagy as a Cellular Stress Response Mechanism in the Nervous System, J Mol Biol 432 (2020) 2560-2588. 10.1016/j.jmb.2020.01.017.

[38] E.K. Fredrickson, R.G. Gardner, Selective destruction of abnormal proteins by ubiquitin-mediated protein quality control degradation, Semin Cell Dev Biol 23 (2012) 530-537. 10.1016/j.semcdb.2011.12.006.

[39] M.S. Uddin, M.A. Rahman, M.T. Kabir, T. Behl, B. Mathew, A. Perveen, G.E. Barreto, M.N. Bin-Jumah, M.M. Abdel-Daim, G.M. Ashraf, Multifarious roles of mTOR signaling in cognitive aging and cerebrovascular dysfunction of Alzheimer's disease, IUBMB Life (2020). 10.1002/iub.2324.

[40] A.S. Dossou, A. Basu, The Emerging Roles of mTORC1 in Macromanaging Autophagy, Cancers (Basel) 11 (2019). 10.3390/cancers11101422.

[41] M.A. Rahman, K. Bishayee, A. Sadra, S.O. Huh, Oxyresveratrol activates parallel apoptotic and autophagic cell death pathways in neuroblastoma cells, Biochim Biophys Acta Gen Subj 1861 (2017) 2336. 10.1016/j.bbagen.2016.10.025.

[42] S. Shaid, C.H. Brandts, H. Serve, I. Dikic, Ubiquitination and selective autophagy, Cell Death Differ 20 (2013) 21-30. 10.1038/cdd.2012.72.

[43] M. Lippai, P. Low, The role of the selective adaptor p62 and ubiquitin-like proteins in autophagy, Biomed Res Int 2014 (2014) 832704. 10.1155/2014/832704.

[44] W. Fan, Z. Tang, D. Chen, D. Moughon, X. Ding, S. Chen, M. Zhu, Q. Zhong, Keap1 facilitates p62mediated ubiquitin aggregate clearance via autophagy, Autophagy 6 (2010) 614-621. 10.4161/auto.6.5.12189.

[45] M.A. Rahman, H. Hwang, Y. Cho, H. Rhim, Modulation of O-GlcNAcylation Regulates Autophagy in Cortical Astrocytes, Oxid Med Cell Longev 2019 (2019) 6279313. 10.1155/2019/6279313.

[46] M.A. Islam, M.A. Sooro, P.H. Zhang, Autophagic Regulation of p62 is Critical for Cancer Therapy, International Journal of Molecular Sciences 19 (2018). ARTN 1405

10.3390/ijms19051405.

[47] Z.Y. You, W.X. Jiang, L.Y. Qin, Z. Gong, W. Wan, J. Li, Y.S. Wang, H.T. Zhang, C. Peng, T.H. Zhou, C. Tang, $W$. Liu, Requirement for $p 62$ acetylation in the aggregation of ubiquitylated proteins under nutrient stress, Nat Commun 10 (2019). ARTN 5792

10.1038/s41467-019-13718-w.

[48] P.C. Trivedi, J.J. Bartlett, T. Pulinilkunnil, Lysosomal Biology and Function: Modern View of Cellular Debris Bin, Cells-Basel 9 (2020). 10.3390/cells9051131. 
[49] I. Eriksson, P. Waster, K. Ollinger, Restoration of lysosomal function after damage is accompanied by recycling of lysosomal membrane proteins, Cell Death Dis 11 (2020). ARTN 370

10.1038/s41419-020-2527-8.

[50] H.K. Song, A. Orr, M.T. Duan, A.J. Merz, W. Wickner, Sec17/Sec18 act twice, enhancing membrane fusion and then disassembling cis-SNARE complexes, Elife 6 (2017). ARTN e26646

10.7554/eLife.26646.

[51] A. Steinauer, J.R. LaRochelle, S.L. Knox, R.F. Wissner, S. Berry, A. Schepartz, HOPS-dependent endosomal fusion required for efficient cytosolic delivery of therapeutic peptides and small proteins, $\mathrm{P}$ Natl Acad Sci USA 116 (2019) 512-521. 10.1073/pnas.1812044116.

[52] U. Nair, A. Jotwani, J.F. Geng, N. Gammoh, D. Richerson, W.L. Yen, J. Griffith, S. Nag, K. Wang, T. Moss, M. Baba, J.A. Mcnew, X.J. Jiang, F. Reggiori, T.J. Melia, D.J. Klionsky, SNARE Proteins Are Required for Macroautophagy, Cell 146 (2011) 290-302. 10.1016/j.cell.2011.06.022.

[53] S. Vats, R. Manjithaya, A reversible autophagy inhibitor blocks autophagosome-lysosome fusion by preventing Stx17 loading onto autophagosomes, Mol Biol Cell 30 (2019) 2283-2295. 10.1091/mbc.E1808-0482.

[54] S. Aivazidis, A. Jain, A.K. Rauniyar, C.C. Anderson, J.O. Marentette, D.J. Orlicky, K.S. Fritz, P.S. Harris, D. Siegel, K.N. Maclean, J.R. Roede, SNARE proteins rescue impaired autophagic flux in Down syndrome, Plos One 14 (2019). ARTN e0223254

10.1371/journal.pone.0223254.

[55] K. Arasaki, H. Nagashima, Y. Kurosawa, H. Kimura, N. Nishida, N. Dohmae, A. Yamamoto, S. Yanagi, Y. Wakana, H. Inoue, M. Tagaya, MAP1B-LC1 prevents autophagosome formation by linking syntaxin 17 to microtubules, Embo Rep 19 (2018). ARTN e45584

10.15252/embr.201745584.

[56] A. Danieli, S. Martens, p62-mediated phase separation at the intersection of the ubiquitinproteasome system and autophagy, J Cell Sci 131 (2018). UNSP jcs214304

$10.1242 /$ jcs.214304.

[57] Y.G. Zhao, H. Zhang, Autophagosome maturation: An epic journey from the ER to lysosomes, Journal of Cell Biology 218 (2019) 757-770. 10.1083/jcb.201810099.

[58] L. Ge, D. Melville, M. Zhang, R. Schekman, The ER-Golgi intermediate compartment is a key membrane source for the LC3 lipidation step of autophagosome biogenesis, Elife 2 (2013). ARTN e00947

10.7554/eLife.00947.

[59] T. Hara, K. Nakamura, M. Matsui, A. Yamamoto, Y. Nakahara, R. Suzuki-Migishima, M. Yokoyama, K. Mishima, I. Saito, H. Okano, N. Mizushima, Suppression of basal autophagy in neural cells causes neurodegenerative disease in mice, Nature 441 (2006) 885-889. 10.1038/nature04724.

[60] A. Metaxakis, C. Ploumi, N. Tavernarakis, Autophagy in Age-Associated Neurodegeneration, CellsBasel 7 (2018). ARTN 37

10.3390/cells7050037.

[61] J.H. Son, J.H. Shim, K.H. Kim, J.Y. Ha, J.Y. Han, Neuronal autophagy and neurodegenerative diseases, Exp Mol Med 44 (2012) 89-98. 10.3858/emm.2012.44.2.031.

[62] N.B. Nedelsky, P.K. Todd, J.P. Taylor, Autophagy and the ubiquitin-proteasome system: Collaborators in neuroprotection, Bba-Mol Basis Dis 1782 (2008) 691-699. 10.1016/j.bbadis.2008.10.002. 
[63] R.J. Baranello, K.L. Bharani, V. Padmaraju, N. Chopra, D.K. Lahiri, N.H. Greig, M.A. Pappolla, K. Sambamurti, Amyloid-Beta Protein Clearance and Degradation (ABCD) Pathways and their Role in Alzheimer's Disease, Curr Alzheimer Res 12 (2015) 32-46. Doi 10.2174/1567205012666141218140953.

[64] S. Ma, I.Y. Attarwala, X.Q. Xie, SQSTM1/p62: A Potential Target for Neurodegenerative Disease, ACS Chem Neurosci 10 (2019) 2094-2114. 10.1021/acschemneuro.8b00516.

[65] A. Caccamo, E. Ferreira, C. Branca, S. Oddo, p62 improves AD-like pathology by increasing autophagy, Mol Psychiatry 22 (2017) 865-873. 10.1038/mp.2016.139.

[66] Y.F. Du, M.C. Wooten, M.W. Wooten, Oxidative damage to the promoter region of SQSTM1/p62 is common to neurodegenerative disease, Neurobiol Dis 35 (2009) 302-310. 10.1016/j.nbd.2009.05.015.

[67] J.R. Babu, T. Geetha, M.W. Wooten, Sequestosome 1/p62 shuttles polyubiquitinated tau for proteasomal degradation, J Neurochem 94 (2005) 192-203. 10.1111/j.1471-4159.2005.03181.x.

[68] Y. Yang, T.L. Willis, R.W. Button, C.J. Strang, Y.H. Fu, X. Wen, P.R.C. Grayson, T. Evans, R.J. Sipthorpe, S.L. Roberts, B. Hu, J.K. Zhang, B.X. Lu, S.Q. Luo, Cytoplasmic DAXX drives SQSTM1/p62 phase condensation to activate Nrf2-mediated stress response, Nat Commun 10 (2019). ARTN 3759

10.1038/s41467-019-11671-2.

[69] S.F. Ma, I.Y. Attarwala, X.Q. Xie, SQSTM1/p62: A Potential Target for Neurodegenerative Disease, Acs Chemical Neuroscience 10 (2019) 2094-2114. 10.1021/acschemneuro.8b00516.

[70] J.R. Babu, M.L. Seibenhener, J.M. Peng, A.L. Strom, R. Kemppainen, N. Cox, H.N. Zhu, M.C. Wooten, M.T. Diaz-Meco, J. Moscat, M.W. Wooten, Genetic inactivation of p62 leads to accumulation of hyperphosphorylated tau and neurodegeneration, J Neurochem 106 (2008) 107-120. 10.1111/j.14714159.2008.05340.x.

[71] M. Glatigny, S. Moriceau, M. Rivagorda, M. Ramos-Brossier, A.C. Nascimbeni, F. Lante, M.R. Shanley, N. Boudarene, A. Rousseaud, A.K. Friedman, C. Settembre, N. Kuperwasser, G. Friedlander, A. Buisson, E. Morel, P. Codogno, F. Oury, Autophagy Is Required for Memory Formation and Reverses Age-Related Memory Decline, Curr Biol 29 (2019) 435-+. 10.1016/j.cub.2018.12.021.

[72] P.L. McClean, C. Holscher, Liraglutide can reverse memory impairment, synaptic loss and reduce plaque load in aged APP/PS1 mice, a model of Alzheimer's disease, Neuropharmacology 76 (2014) 57-67. 10.1016/j.neuropharm.2013.08.005.

[73] J.J. Kong, L.P. Wan, Y.F. Wang, H. Zhang, W. Zhang, Liraglutide Attenuates A beta 42 Generation in APPswe/SH-SY5Y Cells Through the Regulation of Autophagy, Neuropsych Dis Treat 16 (2020) 1817-1825. 10.2147/Ndt.S260160.

[74] A.L. Lin, W. Zheng, J.J. Halloran, R.R. Burbank, S.A. Hussong, M.J. Hart, M. Javors, Y.Y.I. Shih, E. Muir, R.S. Fonseca, R. Strong, A.G. Richardson, J.D. Lechleiter, P.T. Fox, V. Galvan, Chronic rapamycin restores brain vascular integrity and function through NO synthase activation and improves memory in symptomatic mice modeling Alzheimer's disease, J Cerebr Blood F Met 33 (2013) 1412-1421. 10.1038/jcbfm.2013.82.

[75] S. Ozcelik, G. Fraser, P. Castets, V. Schaeffer, Z. Skachokova, K. Breu, F. Clavaguera, M. Sinnreich, L. Kappos, M. Goedert, M. Tolnay, D.T. Winkler, Rapamycin Attenuates the Progression of Tau Pathology in P301S Tau Transgenic Mice, Plos One 8 (2013). ARTN e62459

10.1371/journal.pone.0062459.

[76] C. Wang, X. Zhang, Z.P. Teng, T. Zhang, Y. Li, Downregulation of PI3K/Akt/mTOR signaling pathway in curcumin-induced autophagy in APP/PS1 double transgenic mice, Eur J Pharmacol 740 (2014) 312-320. 10.1016/j.ejphar.2014.06.051.

[77] E.E. Congdon, J.W. Wu, N. Myeku, Y.H. Figueroa, M. Herman, P.S. Marinec, J.E. Gestwicki, C.A. Dickey, W.H. Yu, K.E. Duff, Methylthioninium chloride (methylene blue) induces autophagy and attenuates tauopathy in vitro and in vivo, Autophagy 8 (2012) 609-622. 10.4161/auto.19048. 
[78] I. Luccarini, C. Grossi, S. Rigacci, E. Coppi, A.M. Pugliese, D. Pantano, G. la Marca, T. Ed Dami, A. Berti, M. Stefani, F. Casamenti, Oleuropein aglycone protects against pyroglutamylated-3 amyloid-ss toxicity: biochemical, epigenetic and functional correlates, Neurobiol Aging 36 (2015) 648-663. 10.1016/j.neurobiolaging.2014.08.029.

[79] X.L. Wu, J. Kosaraju, W. Zhou, K.Y. Tam, Neuroprotective Effect of SLM, a Novel Carbazole-Based Fluorophore, on SH-SY5Y Cell Model and 3xTg-AD Mouse Model of Alzheimer's Disease, Acs Chemical Neuroscience 8 (2017) 676-685.

[80] M. Holubova, L. Hruba, A. Popelova, M. Bencze, V. Prazienkova, S. Gengler, H. Kratochvilova, M. Haluzik, B. Zelezna, J. Kunes, C. Holscher, L. Maletinska, Liraglutide and a lipidized analog of prolactinreleasing peptide show neuroprotective effects in a mouse model of beta-amyloid pathology, Neuropharmacology 144 (2019) 377-387. 10.1016/j.neuropharm.2018.11.002.

[81] S. Kim, K.J. Choi, S.J. Cho, S.M. Yun, J.P. Jeon, Y.H. Koh, J. Song, G.V.W. Johnson, C. Jo, Fisetin stimulates autophagic degradation of phosphorylated tau via the activation of TFEB and Nrf2 transcription factors, Sci Rep-Uk 6 (2016).

[82] H.L. Song, A.V. Demirev, N.Y. Kim, D.H. Kim, S.Y. Yoon, Ouabain activates transcription factor EB and exerts neuroprotection in models of Alzheimer's disease, Mol Cell Neurosci 95 (2019) 13-24. 10.1016/j.mcn.2018.12.007.

[83] S. Chandra, M. Jana, K. Pahan, Aspirin Induces Lysosomal Biogenesis and Attenuates Amyloid Plaque Pathology in a Mouse Model of Alzheimer's Disease via PPAR alpha, Journal of Neuroscience 38 (2018) 6682-6699.

[84] S. Chandra, A. Roy, M. Jana, K. Pahan, Cinnamic acid activates PPARalpha to stimulate Lysosomal biogenesis and lower Amyloid plaque pathology in an Alzheimer's disease mouse model, Neurobiol Dis 124 (2019) 379-395. 10.1016/j.nbd.2018.12.007.

[85] U. Kruger, Y.P. Wang, S. Kumar, E.M. Mandelkow, Autophagic degradation of tau in primary neurons and its enhancement by trehalose, Neurobiology of Aging 33 (2012) 2291-2305. 10.1016/j.neurobiolaging.2011.11.009.

[86] S.D. Portbury, D.J. Hare, C. Sgambelloni, K. Perronnes, A.J. Portbury, D.I. Finkelstein, P.A. Adlard, Trehalose Improves Cognition in the Transgenic Tg2576 Mouse Model of Alzheimer's Disease, J Alzheimers Dis 60 (2017) 549-560. 10.3233/Jad-170322.

[87] Y. Li, M. Xu, X. Ding, C. Yan, Z.Q. Song, L.W. Chen, X.H. Huang, X. Wang, Y.L. Jian, G.H. Tang, C.Y. Tang, Y.T. Di, S.Z. Mu, X.Z. Liu, K. Liu, T. Li, Y.C. Wang, L. Miao, W.X. Guo, X.J. Hao, C.L. Yang, Protein kinase C controls lysosome biogenesis independently of mTORC1, Nat Cell Biol 18 (2016) 1065-1077.

[88] T. Jiang, J.T. Yu, X.C. Zhu, Q.Q. Zhang, L. Cao, H.F. Wang, M.S. Tan, Q. Gao, H. Qin, Y.D. Zhang, L. Tan, Temsirolimus attenuates tauopathy in vitro and in vivo by targeting tau hyperphosphorylation and autophagic clearance, Neuropharmacology 85 (2014) 121-130. 10.1016/j.neuropharm.2014.05.032.

[89] L. Lesire, L. Chaput, P.C. De Casas, F. Rousseau, C. Piveteau, J. Dumont, D. Pointu, B. Deprez, F. Leroux, High-Throughput Image-Based Aggresome Quantification, Slas Discov (2020). Artn 2472555220919708

$10.1177 / 2472555220919708$.

[90] J. Schmidt, M.R.G. Marques, S. Botti, M.A.L. Marques, Recent advances and applications of machine learning in solid-state materials science, Npj Comput Mater 5 (2019). ARTN 83

10.1038/s41524-019-0221-0.

[91] J.A. Olzmann, L. Li, L.S. Chin, Aggresome formation and neurodegenerative diseases: Therapeutic implications, Curr Med Chem 15 (2008) 47-60.

[92] J.P. Taylor, F. Tanaka, J. Robitschek, C.M. Sandoval, A. Taye, S. Markovic-Plese, K.H. Fischbeck, Aggresomes protect cells by enhancing the degradation of toxic polyglutamine-containing protein, Hum Mol Genet 12 (2003) 749-757. 10.1093/hmg/ddg074. 
[93] M.S. Uddin, A.A. Mamun, M. Jakaria, S. Thangapandiyan, J. Ahmad, M.A. Rahman, B. Mathew, M.M. Abdel-Daim, L. Aleya, Emerging promise of sulforaphane-mediated Nrf2 signaling cascade against neurological disorders, Sci Total Environ 707 (2020) 135624. 10.1016/j.scitotenv.2019.135624.

[94] M.S. Uddin, A. Al Mamun, M.A. Rahman, T. Behl, A. Perveen, A. Hafeez, M.N. Bin-Jumah, M.M. AbdelDaim, G.M. Ashraf, Emerging Proof of Protein Misfolding and Interaction in Multifactorial Alzheimer's Disease, Curr Top Med Chem (2020). 10.2174/1568026620666200601161703.

[95] L. Ding, W.H. Nan, X.B. Zhu, X.M. Li, L.Y. Zhou, H.J. Chen, L. Yu, F.U. Khan, H.B. Zhong, X.J. Shi, Rapamycin and FK506 derivative TH2849 could ameliorate neurodegenerative diseases through autophagy with low immunosuppressive effect, Cns Neurosci Ther 25 (2019) 452-464. 10.1111/cns.13062. [96] J. Liu, L. Li, Targeting Autophagy for the Treatment of Alzheimer's Disease: Challenges and Opportunities, Front Mol Neurosci 12 (2019). ARTN 203

10.3389/fnmol.2019.00203.

[97] Z. Cai, B. Zhao, K.S. Li, L.Q. Zhang, C.H. Li, S.H. Quazi, Y. Tan, Mammalian target of rapamycin: A valid therapeutic target through the autophagy pathway for alzheimer's disease?, J Neurosci Res 90 (2012) 1105-1118. 10.1002/jnr.23011.

[98] Y.C. Xie, R. Kang, X.F. Sun, M.Z. Zhong, J. Huang, D.J. Klionsky, D.L. Tang, Posttranslational modification of autophagy-related proteins in macroautophagy, Autophagy 11 (2015) 28-45. 10.4161/15548627.2014.984267.

[99] M. Cosenza, S. Pozzi, The Therapeutic Strategy of HDAC6 Inhibitors in Lymphoproliferative Disease, Int J Mol Sci 19 (2018). 10.3390/ijms19082337.

[100] D.C. Rubinsztein, P. Codogno, B. Levine, Autophagy modulation as a potential therapeutic target for diverse diseases, Nat Rev Drug Discov 11 (2012) 709-U784. 10.1038/nrd3802. 